\title{
Engineered silicon carbide three-dimensional frameworks through DNA-prescribed assembly
}

\author{
Aaron Michelson ${ }^{1}$, Honghu Zhang ${ }^{2}$, Shuting Xiang ${ }^{3}$, Oleg Gang ${ }^{1,2,3 *}$ \\ ${ }^{1}$ Department of Applied Physics and Applied Mathematics, Columbia University, New York, \\ NY, 10027 USA \\ ${ }^{2}$ Center for Functional Nanomaterials, Brookhaven National Laboratory, Upton, NY, 11973 USA \\ ${ }^{3}$ Department of Chemical Engineering, Columbia University, New York, NY, 10027 USA
}

\section{$\underline{\text { Supplemental Material }}$}

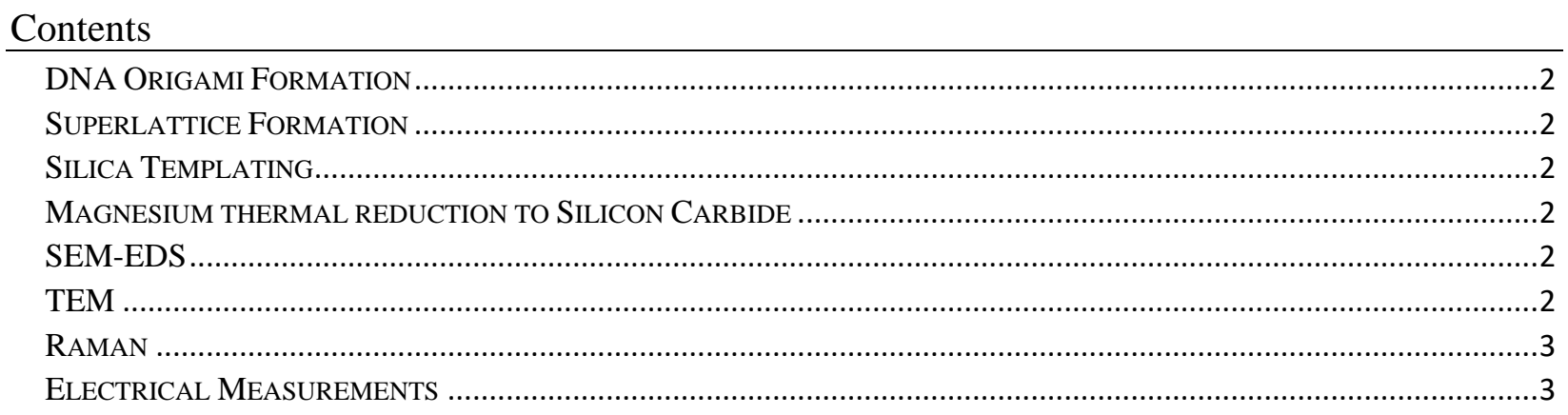

S1. SMALL ANGLE X-RAY SCATTERING (SAXS) AND SCANNING ELECTRON MICROSCOPY - BEFORE CONVERSION 4

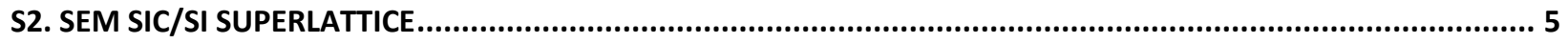





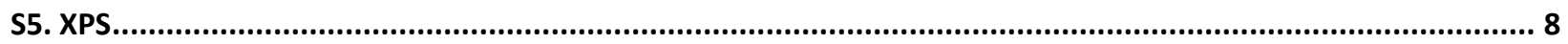

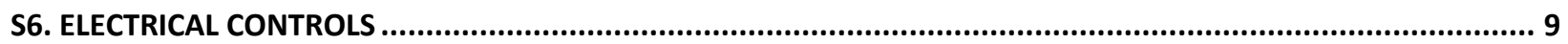

S7. SELECT AREA DIFFRACTION SMOOTHING ................................................................................10

S8. CROSS SECTIONAL TEM AND SCANNING TRANSMISSION MICROSCOPY WITH EDS.................................11

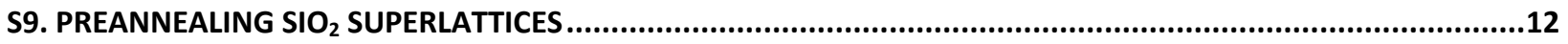

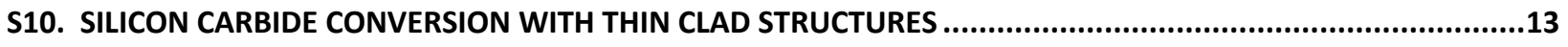

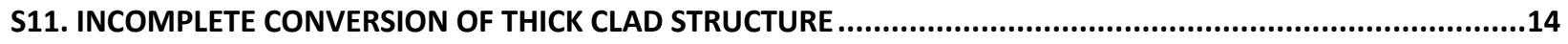


Experimental /Material and Methods:

DNA Origami Formation

DNA origami was formed by mixing 40nm m13mp18 scaffold (Bayou Biolabs) in $1 \times$

TAE and $12.5 \mathrm{mM}$ Magnesium with $10 \times$ concentration of DNA staples, with designs as reported previously ${ }^{1}$. DNA was purchased at regular desalting from ITD.

Origami was annealed using a 20-hour protocol, from a short $90{ }^{\circ} \mathrm{C}$ denaturing step with a slow cooling to room temperature (RT).

Superlattice Formation

Superlattices were formed using $15 \mathrm{ul}$ of $40 \mathrm{nM}$ origami and its complement origami, forming superlattices with an edge length between 1-10um after annealing superlattices from $50^{\circ} \mathrm{C}$ to RT over 4 days with a rate of $-0.1^{\circ} \mathrm{C} / \mathrm{hr}$.

Silica Templating

Superlattices in solution were centrifuged and buffer-exchanged with $1 \times$ Tris and $10 \mathrm{mM}$ Mg. A solution of 0.5\% 3-aminopropyltriethoxysilane (APTES) and 3\% tetraethyl orthosilicate (TEOS) were premixed in $1 \times$ Tris and $10 \mathrm{mM} \mathrm{Mg}$ for 25 minutes at $700 \mathrm{rpm}$ shaking. The solution was filtered using Millex-GV PVDF $0.22 \mathrm{um}$ um filters. $15 \mathrm{ul}$ of filtered solution was mixed with $5 \mathrm{ul}$ of superlattice and put back into a shaker at $700 \mathrm{rpm}$ for 1.5 hours. The reaction was stopped by adding DI water and buffer exchanging the supernatant 2 times with DI water.

The Silica-Superlattice conjugate was drop cast to a silicon chip substrate for observation in SEM.

Magnesium thermal reduction to Silicon Carbide

A 2ul drop of concentrated lattice was drop cast to either tungsten or molybdenum foil; this was then placed into a molybdenum boat with 5-15mg Mg turnings, and covered and placed into a tube furnace (Lindberg CVD furnace). The tube furnace was evacuated to $400 \mathrm{mtorr}$, and backfilled with $\mathrm{Ar} / 2 \% \mathrm{H} 2$ gas to an initial pressure of 9 torr. The furnace was then closed from both ends and the temperature ramped to $660^{\circ} \mathrm{C}$ and held for 3 hours. The sample was removed from the furnace after cooling down near RT. The sample substrate was submerged in $2 \mathrm{M} \mathrm{HCl}$ for $12-24$ hours to removed $\mathrm{MgO}$ on the surface of the sample, washed with DI water and dried with nitrogen, imaged or additionally submerged in HF 10:1 Buffered oxide etch for 5-10 minutes to remove excess $\mathrm{SiO}_{2}$ surface oxide. The resulting sample was blow dried with nitrogen.

\section{SEM-EDS}

Electron microscopy was performed using a Jeol $7600 \mathrm{~F}$, operated between $5-15 \mathrm{keV}$ to probe surface and deeper volumes of the superlattice as needed. EDS spectrum were collected for superlattice areas using an Oxford $80 \mathrm{~mm}$ detector for at least 100k counts.

TEM

Transmission electron microscopy was performed using a Jeol 1400 operated at 120 $\mathrm{KeV}$. For high resolution scans an FEI Talos operated at $200 \mathrm{KeV}$ for EDS, and a Jeol 
2100 at $200 \mathrm{KeV}$ for eds/high resolution microscopy were used. SAED patterns were collected from all equipment.

Raman

Raman was performed with the Witec Alpha probe with a 532nm laser operated at room temperature.

Electrical Measurements:

In-Situ FIB with a Keithley operating on a custom lab-view code used to generate current up to $1 \mathrm{~mA}$ with a voltage sweep from -/+10 Volts. The omniprobe was used as one probe with the stage ground connected to the ground terminal on the supply.

Small Angle X-ray Scattering

Small angle x-ray scattering (SAXS) was performed at the Complex Materials Scattering (CMS) beamline at the National Synchroton Light Source II (NSLSII). The beam had an energy of $13.5 \mathrm{keV}$, and the data were collected using a Pilatus $2 \mathrm{M}$ detector, placed 5 meters downstream. 
S1. Small Angle X-Ray Scattering (SAXS) and Scanning Electron Microscopy - Before Conversion
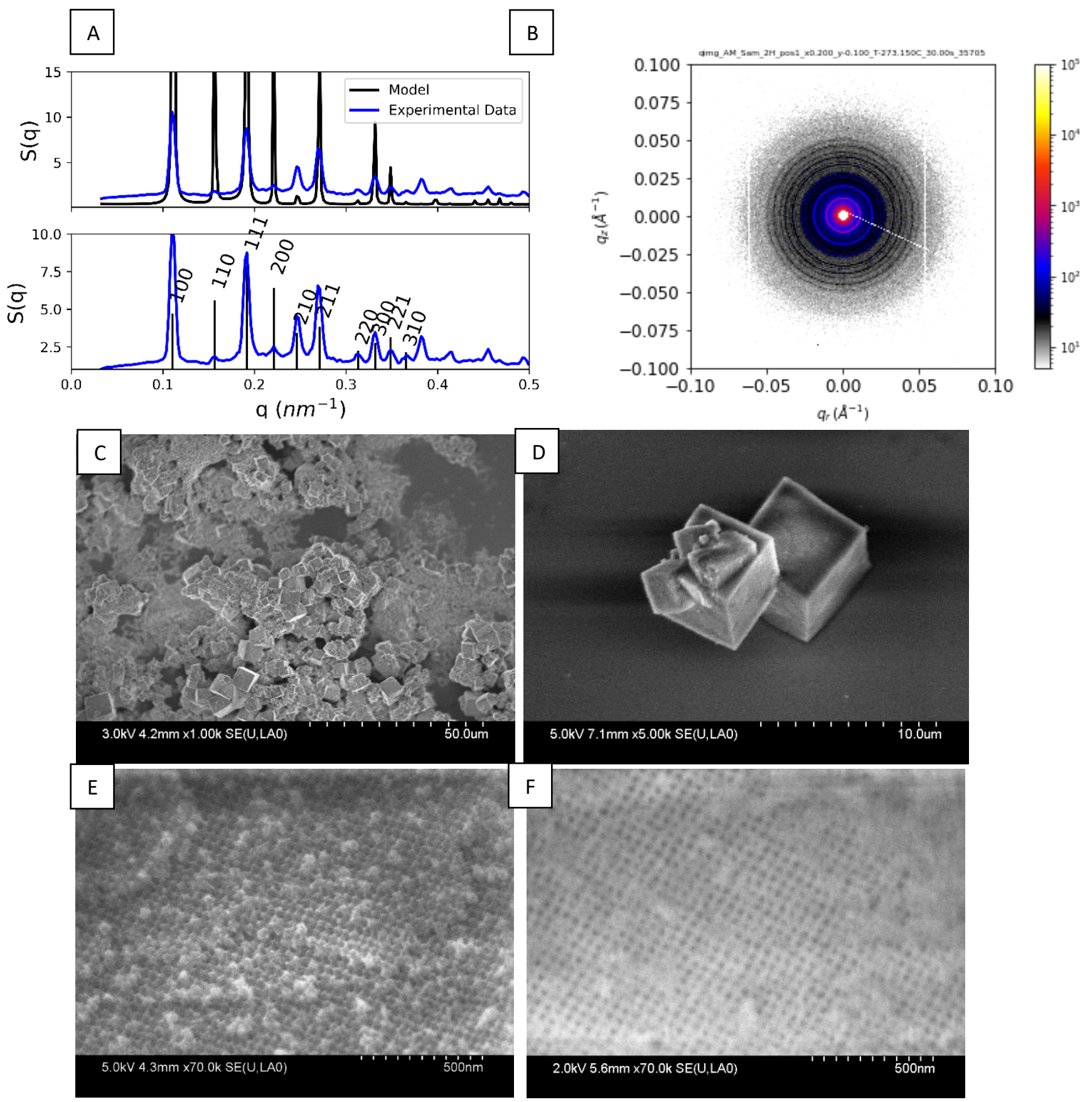

Figure S1. SAXS of DNA-superlattice and SEM of Silica-Superlattices

a) Experimental structure factor, $\mathrm{S}(\mathrm{q})$, as obtained from SAXS measurements, for a DNA superlattice in solution (blue), and a modelled ${ }^{1}$ scattering of the corresponding simple cubic lattice (black) are shown in the top panel; the first 10 peaks are indexed in the lower panel. b) 
Image of x-ray scattering of the structure. c) SEM of silicated DNA origami superlattices before conversion to silicon-carbide/silicon. The samples range in size from $<1$ um to over 10um from agglomerated to singular domains. The pore size can be visualized in figures e, and $\mathrm{f}$ to be $15-20 \mathrm{~nm}$.

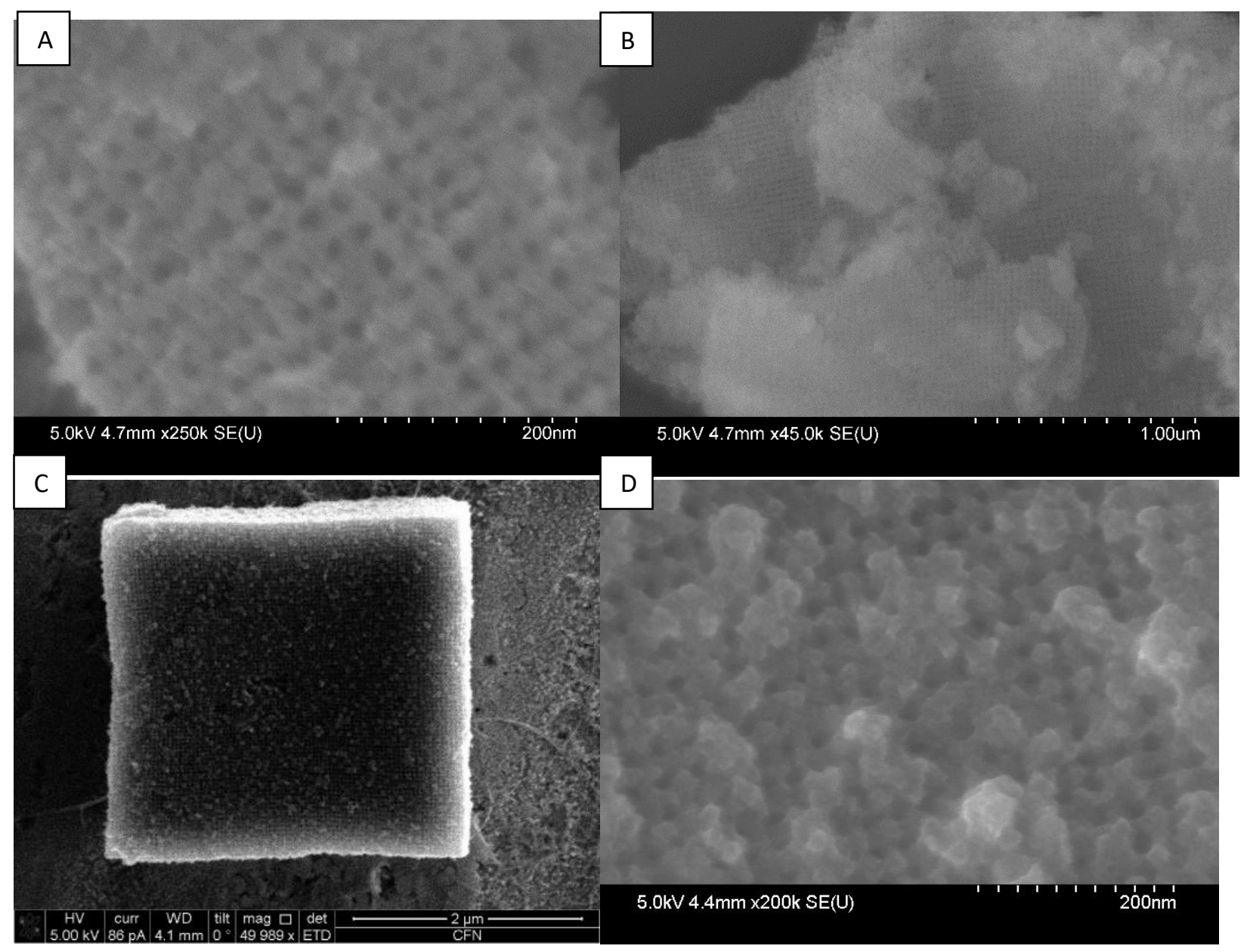

Figure S2. SEM of SiC samples at various magnifications (A-D)

SEM of samples after magnesio-thermal reduction. Both quasi-2d and 3D superlattices demonstrate sharp features of the underlying lattice with pore sizes in the similar 10-20nm range of the pre-silicated superlattices, the strut diameter can be viewed in both A and D between 8$20 \mathrm{~nm}$. 


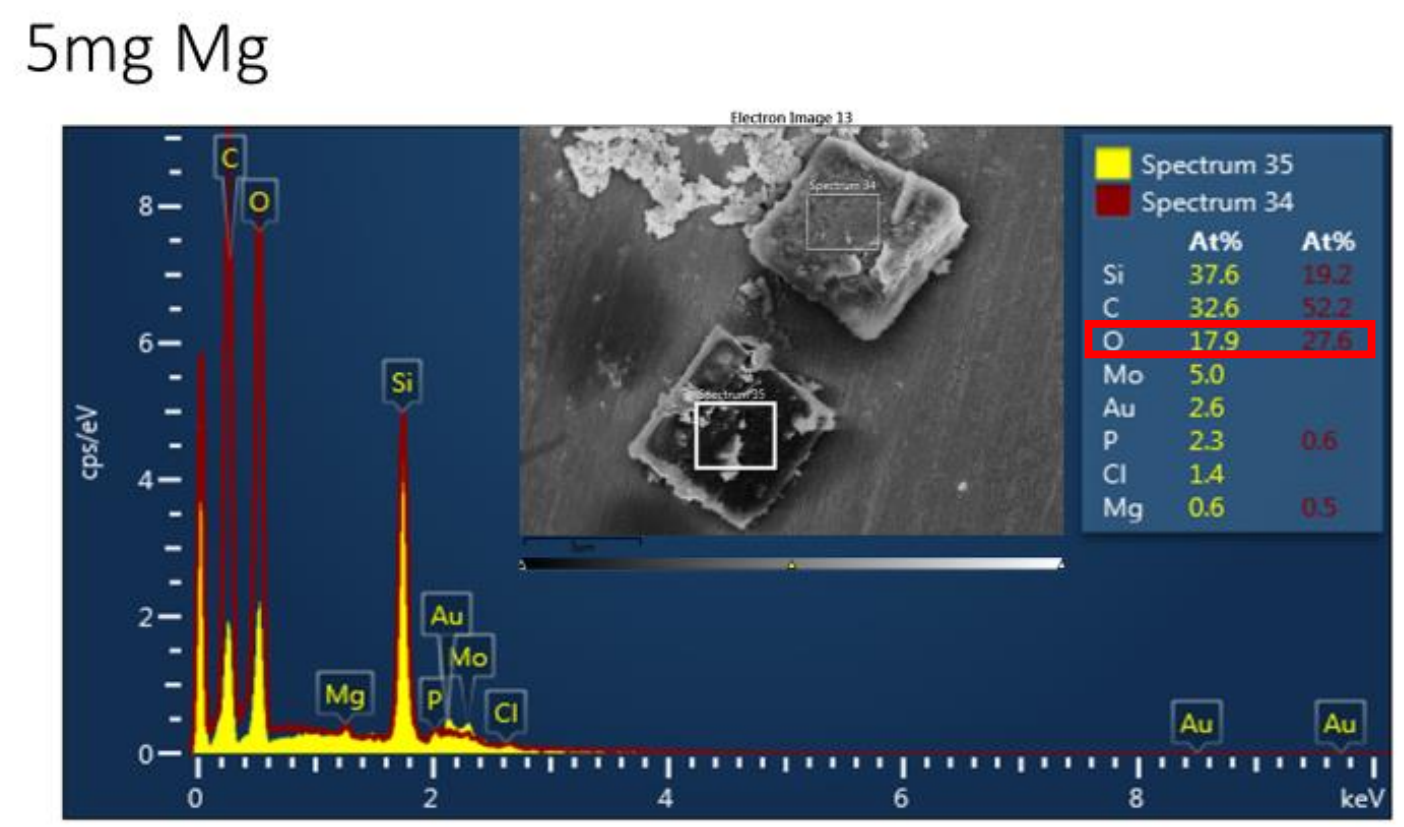

Figure S3. 5mg Mg - Incomplete conversion to SiC. EDS from the sample shows a large difference in the oxygen signal (27 at\% $\mathrm{O}$ in $\mathrm{SiO} 2$ superlattice vs 17 at\% in partial $\mathrm{SiC}$ sample) strongly suggesting that one is largely Silica vs Silicon Carbide. This incomplete conversion is likely due to an insufficient amount of magnesium in the reaction chamber. 

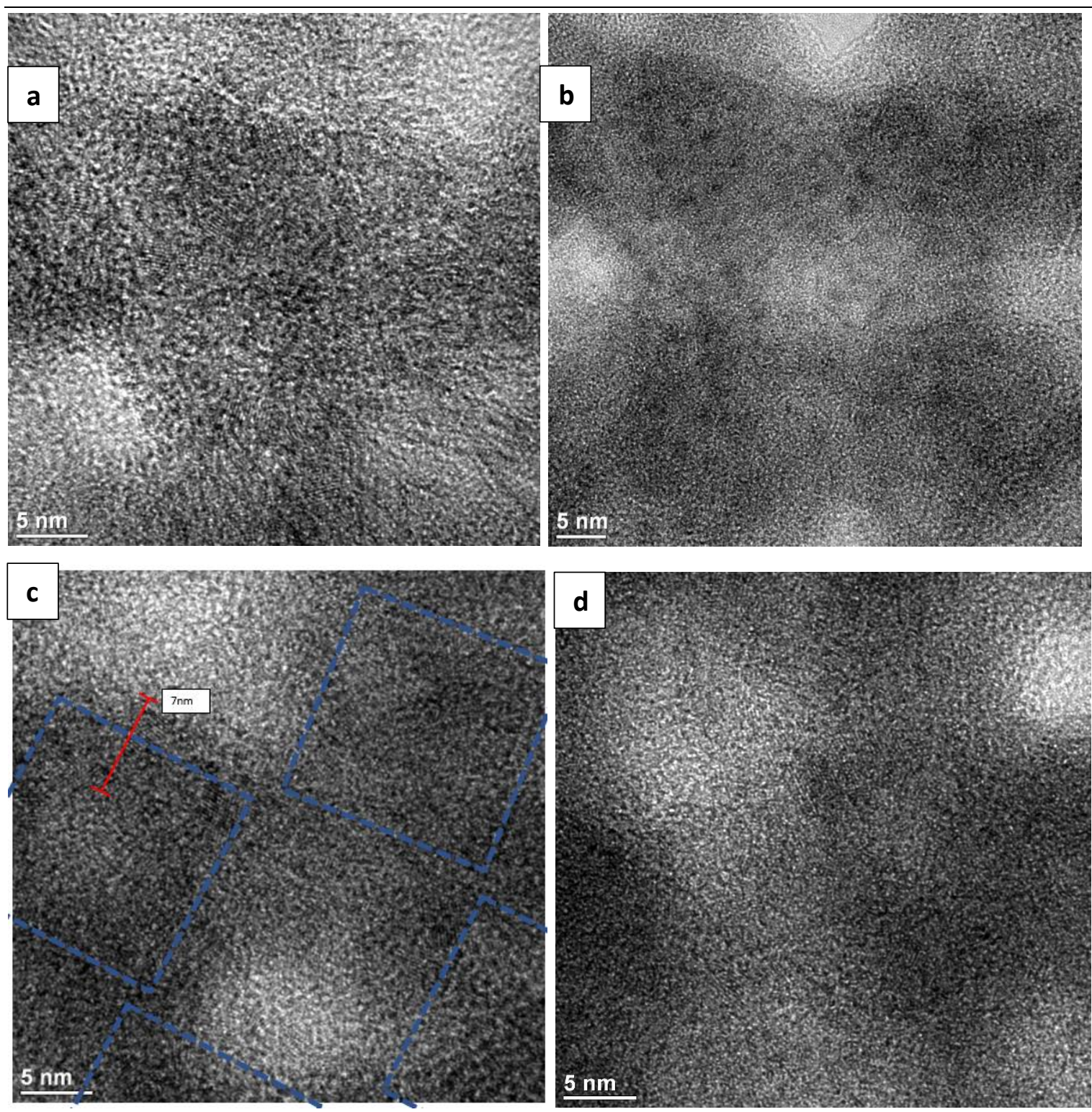

Figure S4. a) High resolution images of the $\mathrm{SiC}$ domains within the superlattice. b)Octahedron (black) arranged in a 2x2 array section of the superlattice c) A section of superlattice wherein large white areas are blank space between origami and the more subtle white space is the interior void of the origami superlattice. The strut measures approximately $7 \mathrm{~nm}$ in diameter d) Zoomed in area of nearby region 


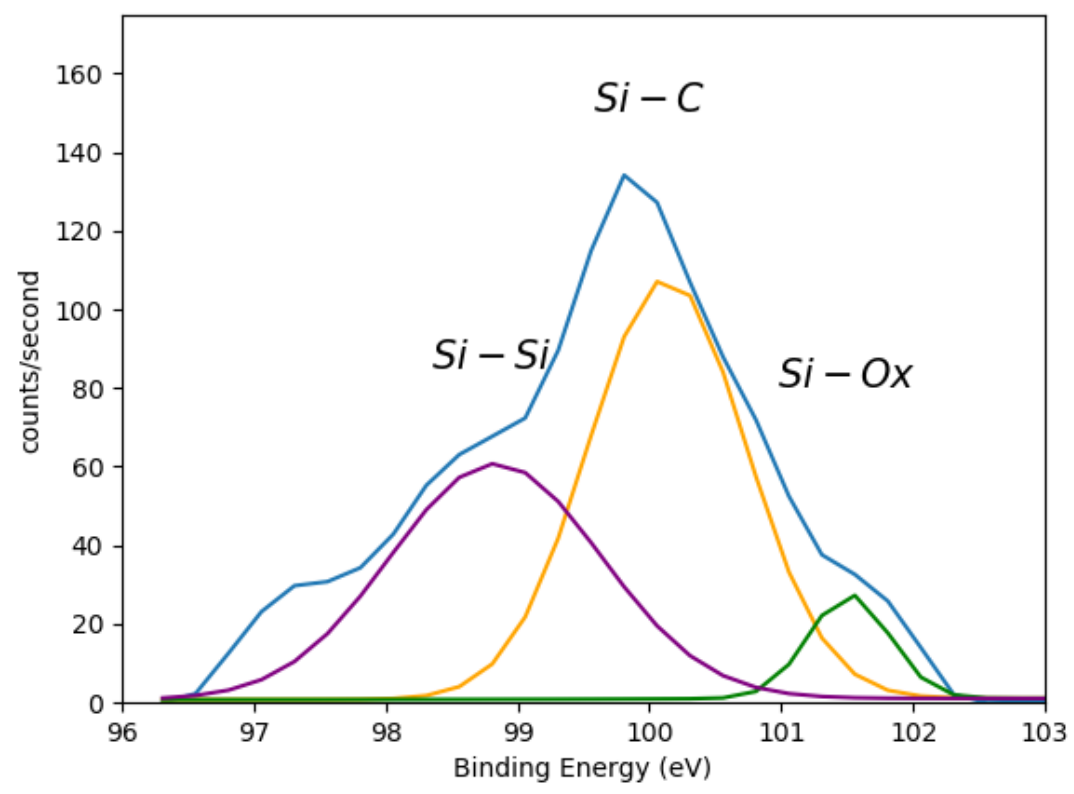

\begin{tabular}{|l|r|r|r|r|r|r|r|r|}
\hline Band & Pos & PosSep & B_FWHM & FWHM & Height & \% Gauss & Area & \% Area \\
\hline $\begin{array}{l}\text { 1(Si- } \\
\text { Si) }\end{array}$ & 98.48 & 0 & 1.47 & 1.47 & 66 & 90 & 108 & $\mathbf{3 3 . 5 6}$ \\
\hline $\begin{array}{l}\mathbf{2}(\mathbf{S i}- \\
\text { C) }\end{array}$ & 100.12 & 1.65 & 1.51 & 1.51 & 123 & 100 & 197 & $\mathbf{6 1 . 2 4}$ \\
\hline $\begin{array}{l}\mathbf{3}(\mathbf{S i}- \\
\mathbf{O x})\end{array}$ & 101.55 & 3.07 & 0.66 & 0.66 & 24 & 100 & 17 & $\mathbf{5 . 2}$ \\
\hline
\end{tabular}

Figure S5. XPS fitting of SiC Superlattice.

Peak fitting with Multipak v9 the SiC/Si superlattice shows a broad peak centered around $100 \mathrm{eV}$ (Si-C) $61 \%$. Significant portions of the spectrum fit $\mathrm{Si}-\mathrm{O} 5 \%$ and $\mathrm{Si}-\mathrm{Si} 33 \%$ bonds suggesting the matrix is composed of $\mathrm{SiC}$ crystal, amorphous $\mathrm{Si}$ and some $\mathrm{SiO}_{2}$ which was not overwise removed by HF. 




Figure S6 Control tests of the conductivity of glass, and sol-gel formed silica from the same batch as the superlattice but without DNA. Signal was recorded with a Signatone 


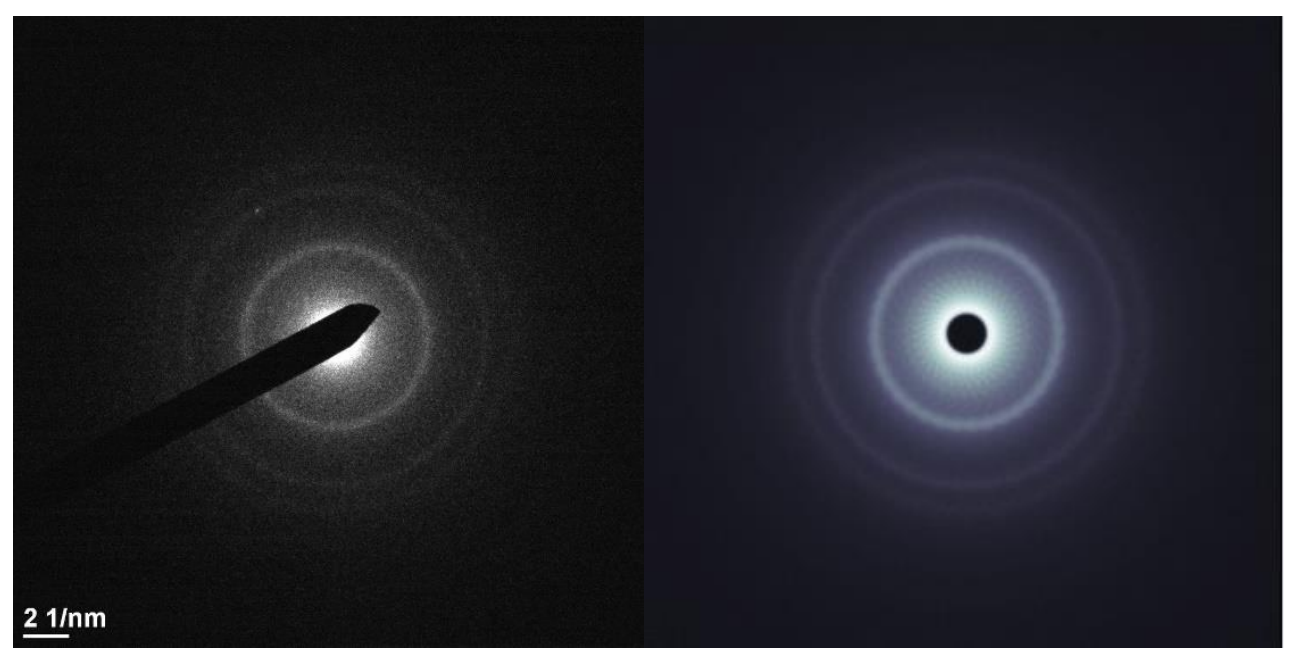

Figure S7. SAED Pattern.

The TEM diffraction pattern was smoothed for visualization purposes by using Scikit-UED, an open source python package for electron diffraction analysis and modeling. Where here the beamstop was removed by fold rotation of the data. 

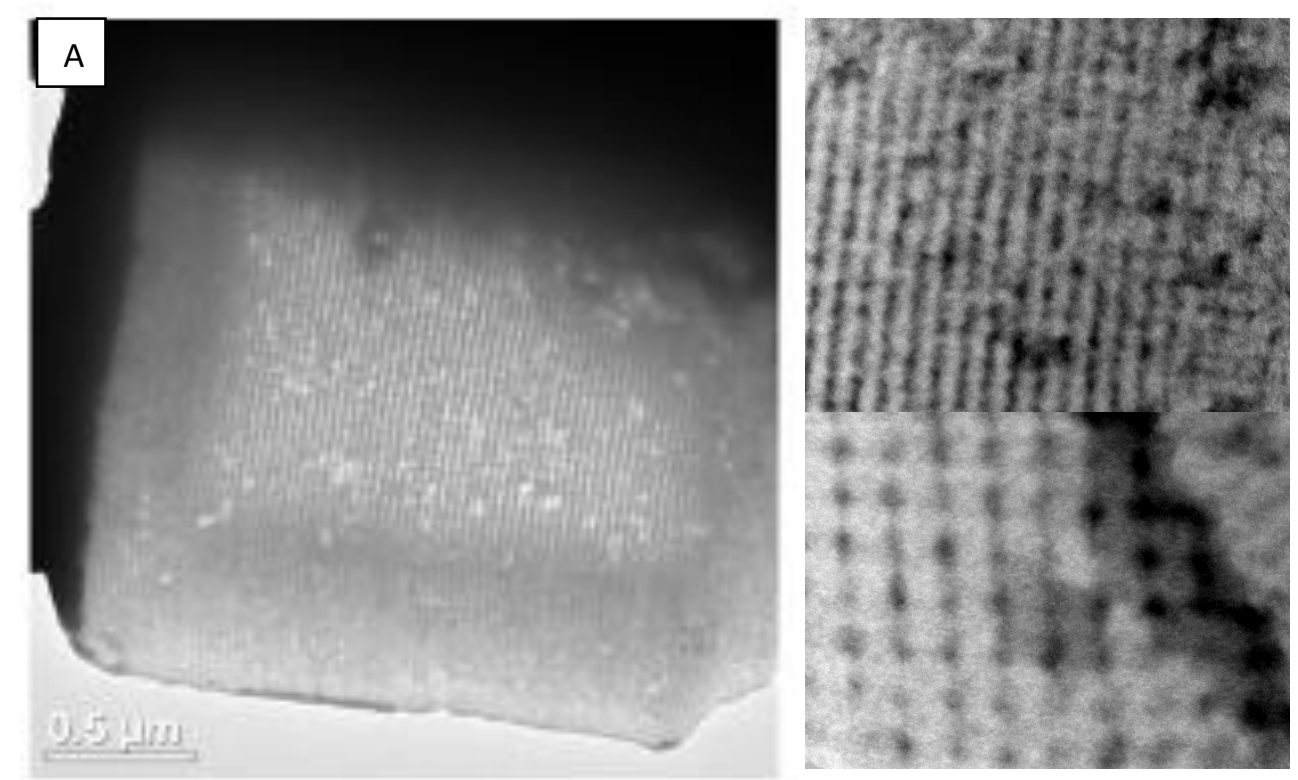

B


Figure S8. Cross Sectional TEM and STEM-EDS

To evaluate the condition of the composition of the superlattice far in the interior of the sample cross section TEM was performed on a superlattice. a) A thin 2-3 layer structure is visible with clear nanostructure visible in the interior of a 3 um superlattice. b) STEM-EDS helps to evaluate the silicon, carbon, and oxygen content of the superlattice structure. Areas show Si and C 
overlapping approximately equal on the intensity scale. (1:1 ratio of Si-C). Oxygen and Phosphorous are contrast adjusted to display features.

\section{Using Preanneal $500 \mathrm{C}$}

Strongly Suggests SiC

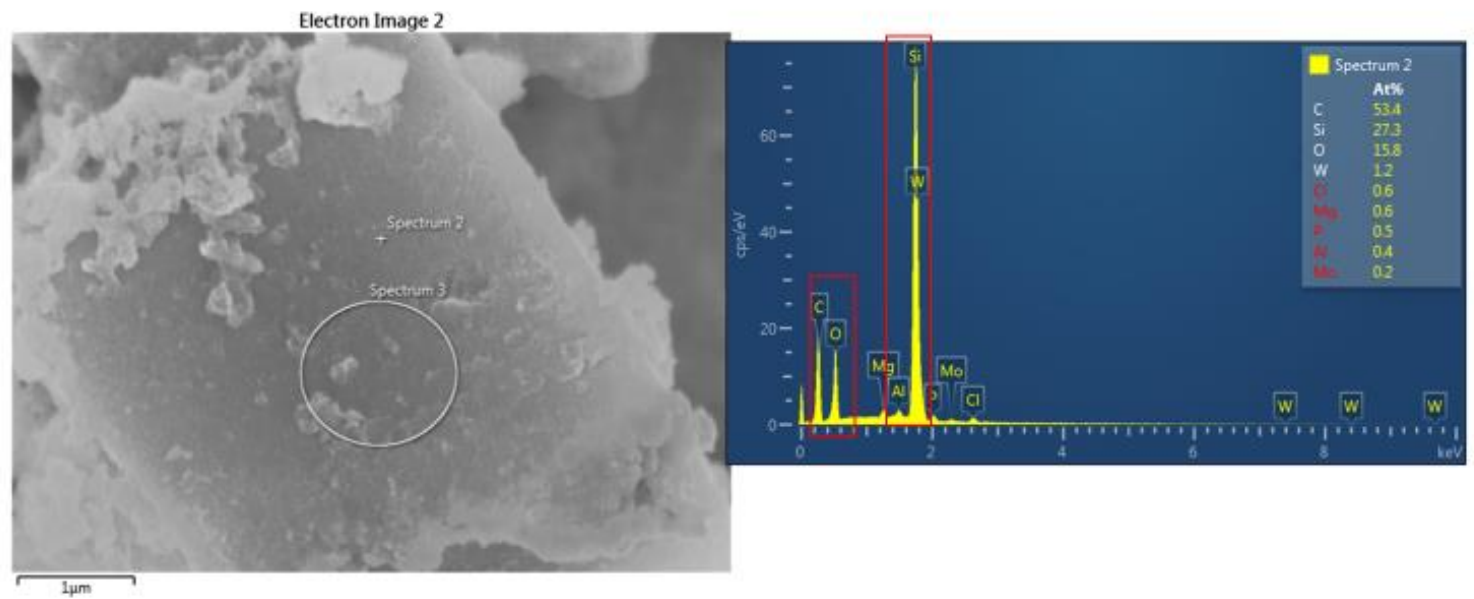

Figure S9. Pre-annealed $\mathrm{SiO}_{2}$ Superlattice post conversion

Courchesne et al. have shown an ability to produce silicon via a pre-annealing step which removed carbon from the bio-templated material. ${ }^{2}$ This seems to have been difficult to do in our case. Where pre-annealing the sample in air at 500C for 3 hours prior to magnesium reduction did not lead to a reduction in carbon signal post conversion. 
S10. Silicon Carbide Conversion with thin clad structures

Thin clad structures (thickness approximately $3-4 \mathrm{~nm}$ ) converted to silicon carbide. As seen in the top panel, some structures that completed conversion and immersion in $\mathrm{HCl} / \mathrm{HF}$ for $\mathrm{Mg}$ removal suffer from partial collapse or significant surface remodeling. However simultaneously numerous structures on the surface demonstrate fine preservation of the superlattice structure retaining nanoscale features. It is unclear at what point structures suffered collapse either due to thin cladding or the vigorous conversion process with magnesium.

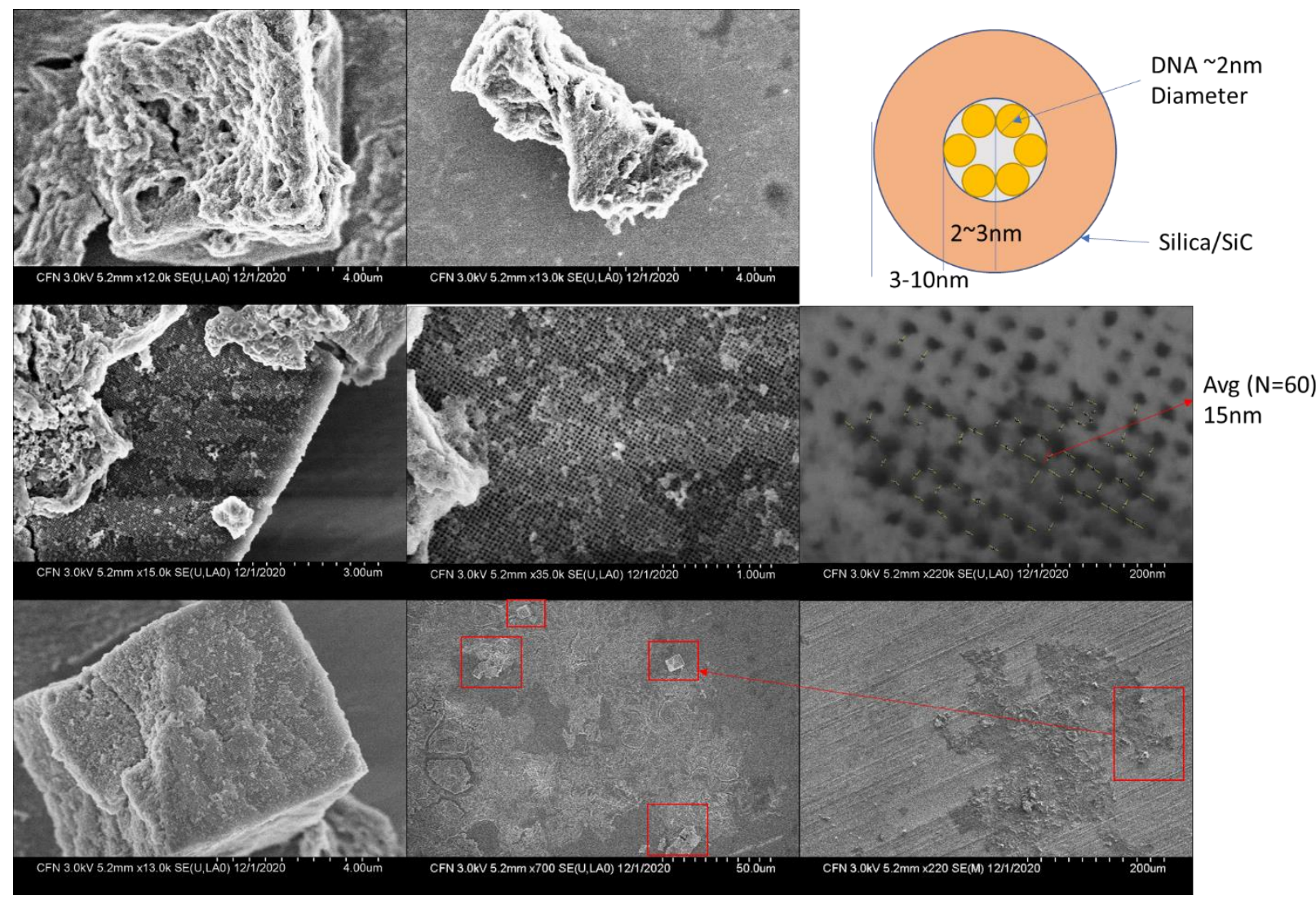

Figure S10. Superlattices with thin cladding with images of outliers that suffered collapse. Top right image is a cross sectional view of the DNA origami strut a 6 hex-bundle of dna with approximate dimensions expected from the diameter of DNA $(2 \mathrm{~nm})$ and visible thickness of the silica cladding. 
TEM cross section of a superlattice converted in magnesium yet displaying no SAED pattern corresponding to crystalline structure. The structure can be seen to have regions of growth in between the layers of origami which might suggest that blocked pathways prevented full conversion of the structure to $\mathrm{SiC}$.

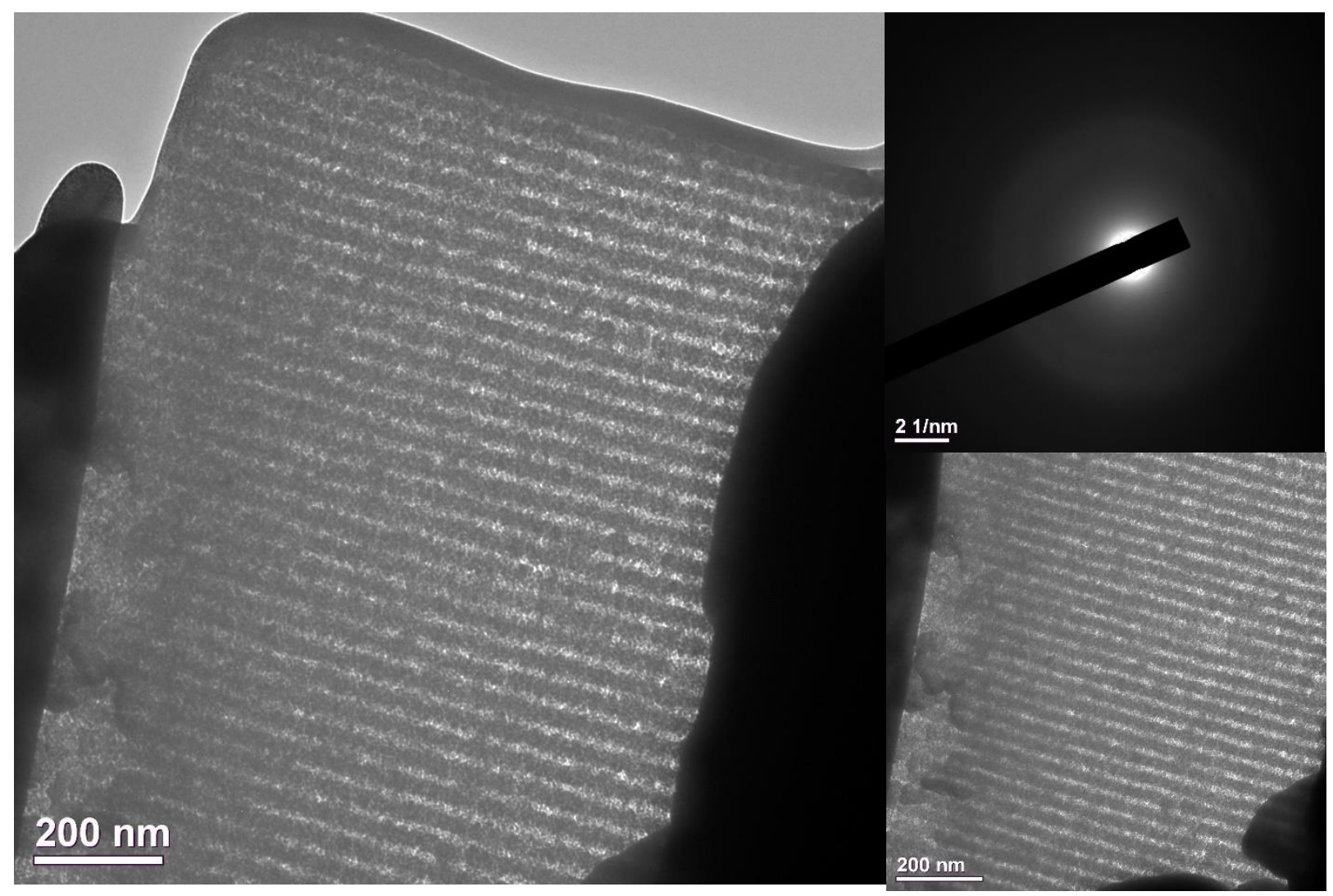

Figure S11. TEM cross-section of sio2 superlattice with incomplete conversion to SiC. A) large top portion of crystal with nanostructure visible as layers of material with silica interconnecting layers at various junctions throughout. B) SAED pattern from the entire region with only a diffuse pattern indicative of amorphous material. C) Lower left region under different focus conditions. 


\section{Citations}

45. Tian, Y., Lhermitte, J. R., Bai, L., Vo, T., Xin, H. L. L., Li, H. L., Li, R. P., Fukuto, M., Yager, K. G., Kahn, J. S., Xiong, Y., Minevich, B., Kumar, S. K. \& Gang, O. Ordered three-dimensional nanomaterials using DNAprescribed and valence-controlled material voxels. Nature Materials, doi:10.1038/s41563-019-0550-x (2020).

25. Dorval Courchesne, N.-M., Steiner III, S. A., Cantú, V. J., Hammond, P. T. \& Belcher, A. M. Biotemplated silica and silicon materials as building blocks for micro-to nanostructures. Chemistry of Materials 27, 536, 1-5370 (2015). 